\title{
Use of Hydroxychloroquine and Risk of Heart Failure in Patients With Rheumatoid Arthritis
}

\author{
Ahmed A. Sorour ${ }^{1}$ (D), Reto D. Kurmann ${ }^{1}$ (D) Youssef E. Shahin $^{1}$, Cynthia S. Crowson ${ }^{2}$ (D), \\ Sara J. Achenbach ${ }^{3}$, Rekha Mankad ${ }^{1}$ (D) and Elena Myasoedova ${ }^{4}$
}

ABSTRACT. Objective. To examine the relationship between the use of hydroxychloroquine (HCQ) and risk of developing heart failure (HF) in rheumatoid arthritis (RA).

Methods. In this nested case-control study, cases were Olmsted County, Minnesota residents with incident RA (based on 1987 American College of Rheumatology criteria) from 1980 to 2013 who developed HF after RA incidence. Each case was matched on year of birth, sex, and year of RA incidence with an RA control who did not develop HF. Data on HCQ use including start and stop dates, as well as dose changes, were reviewed and used to calculate HCQ duration and cumulative dose. Age-adjusted logistic regression models were used to examine the association between HCQ and HF.

Results. The study identified 143 RA cases diagnosed with HF (mean age 65.8 yrs, 62\% females) and 143 non-HF RA controls (mean age 64.5, 62\% female). HCQ cumulative dose was not associated with HF (OR 0.96 per 100-g increase in cumulative dose, 95\% CI 0.90-1.03). Likewise, no association was found for patients with a cumulative dose $\geq 300 \mathrm{~g}$ (OR $0.92,95 \% \mathrm{CI} 0.41-2.08$ ). The HCQ duration of intake in years prior to index was not associated with HF (OR 0.98, 95\% CI 0.91-1.05).

Conclusion. Use of HCQ was not associated with development of HF in patients with RA in this study. Further studies are needed to understand the effect of higher doses of HCQ on the development of HF in RA.

Key Indexing Terms: drug toxicity, heart failure, hydroxychloroquine, rheumatoid arthritis

Rheumatoid arthritis (RA) is a chronic systemic inflammatory disease characterized by joint damage and associated functional disability. ${ }^{1}$ Increased risk of cardiovascular (CV) disease in RA as compared with the general population has been shown in multiple studies. ${ }^{2}$ Further, patients with RA have been shown to have a 2 -fold increased risk of developing congestive heart failure (HF) compared to subjects without RA. ${ }^{3}$

Hydroxychloroquine (HCQ) is a disease-modifying antirheumatic drug that has been widely used for the treatment of RA. ${ }^{4} \mathrm{HCQ}$ cardiotoxicity has been implicated as a rare yet potentially life-threatening side effect of the drug, primarily causing cardiomyopathy that results in $\mathrm{HF}^{5}$ The evidence of

This study was supported by grants from the National Institutes of Health/ National Institute of Arthritis and Musculoskeletal and Skin Diseases (R01 grants: AR046849, AG034676).

${ }^{1}$ A.A. Sorour, MD, R.D. Kurmann, MD, Y.E. Shahin, R. Mankad, MD, Department of Cardiovascular Medicine, Mayo Clinic; ${ }^{2}$ C.S. Crowson, $\mathrm{PhD}$, Division of Biomedical Statistics and Informatics, and Department of Rheumatology, Mayo Clinic; ${ }^{3} S$.J. Achenbach, MS, Division of Biomedical Statistics and Informatics, Mayo Clinic; ${ }^{4}$ E. Myasoedova, MD, PhD, Department of Rheumatology, Mayo Clinic, Rochester, Minnesota, USA.

The authors declare no conflicts of interest.

Address correspondence to Dr. E. Myasoedova, Department of Rheumatology, Mayo Clinic, 200 First St SW, Rochester, MN 55905, USA.

Email:myasoedova.elena@mayo.edu.

Accepted for publication January 8, 2021. cardiotoxicity associated with the use of HCQ relies mainly on case reports and case series, and large cohort studies on the subject are lacking. In this population-based study, we aimed to examine the association between exposure to HCQ and development of HF in patients with RA.

\section{METHODS}

Study design and population. This nested case-control study was conducted using the resources of the Rochester Epidemiology Project (REP). The REP system ensures ready access to all inpatient and outpatient records of Olmsted County, Minnesota residents from all community medical providers including the Mayo Clinic, its affiliated hospitals, and the Olmsted Medical Center. ${ }^{6}$ The study included adult patients with incident RA from the preidentified cohort of all incident cases with RA in Olmsted County from 1980 to 2013. All patients included in the study were $\geq 18$ years of age and fulfilled the 1987 American College of Rheumatology (ACR) classification criteria. ${ }^{7}$ Cases were defined as patients with RA who developed HF after RA incidence (HF cases), whereas controls were RA patients without HF (non-HF controls). Cases and controls were matched 1:1 on year of birth, sex, and year of RA incidence. Each non-HF control was assigned an index date corresponding to the HF diagnosis date of the case, which occurred in 1983-2018. Controls were allowed to later become cases to avoid bias. HF was defined using the Framingham Heart Study criteria. ${ }^{8}$ Information on HCQ-related retinal toxicity was also obtained from the medical records.

HCQ exposure. To determine the history of HCQ use, medical records were manually reviewed and abstracted. Data extracted included the HCQ start date, dosage, change in dosage, stop date, reason for termination, and restart date, if applicable. The glomerular filtration rate (GFR) was calculated from creatinine values obtained closest to index date/HF diagnosis with \pm 90 days using the CKD-EPI (Chronic Kidney Disease Epidemiology 
Collaboration) formula. This study was approved by institutional review boards of Mayo Clinic (IRB \#17-002593) and Olmsted Medical Center (IRB \#017-omc-17).

Data on $C V$ risk factors and $R A$ characteristics. A review of medical records was performed to record the following $C V$ risk factors: age, smoking, hypertension (HTN), diabetes mellitus (DM), and dyslipidemia, as previously defined. ${ }^{9}$ Data on RA disease characteristics such as rheumatoid factor positivity, anticyclic citrullinated peptide antibody positivity, and year of RA diagnosis were collected for all patients.

Statistical analysis. Descriptive statistics (means, percentages) were used to summarize the data. Characteristics were compared between cases and controls using chi-square and rank-sum tests. Age-adjusted logistic regression models were used to examine the association between HCQ and HF. Cumulative dose was examined as a continuous variable and dichotomously (<300 vs $\geq 300 \mathrm{~g}$ ). Analyses were performed using SAS version 9.4 (SAS Institute) and R 3.6.2 (R Foundation for Statistical Computing).

\section{RESULTS}

Patient characteristics. From a cohort of 1078 patients with RA, this study identified 143 RA cases diagnosed with HF who were matched with 143 non-HF RA controls. Table 1 shows characteristics of both groups at RA incidence. Both groups were similar in respect to demographics and RA characteristics. HTN and DM were more prevalent in the HF cases. The median (IQR) GFR $\left(\mathrm{mL} / \mathrm{min} / 1.73 \mathrm{~m}^{2}\right)$ at index date was 66.5 (53.8-78.5) and 58.1 (41.8-74.7) in non-HF controls and HF cases, respectively. The initial presentation of the HF group based on the Framingham criteria is summarized in Table 2.

Duration of HCQ use and risk of HF. Seventy-one HF and 69 non-HF patients used HCQ at some point prior to HF diagnosis/index date. The median (IQR) duration of HCQ use prior to index date was $2.8(0.6-10)$ years in HF cases and 2.5 (0.7-8.2) years in non-HF controls (Table 3). Age-adjusted

Table 1. Characteristics at RA incidence in patients who developed HF during the follow-up and those who did not.

\begin{tabular}{|c|c|c|c|}
\hline & $\begin{array}{c}\text { HF } \\
\mathrm{n}=143\end{array}$ & $\begin{array}{l}\text { Non-HF, } \\
\mathrm{n}=143\end{array}$ & $P$ \\
\hline $\begin{array}{l}\text { Age at RA diagnosis, yrs, } \\
\text { mean } \pm S D\end{array}$ & $65.8 \pm 12.3$ & $64.5 \pm 12.5$ & 0.42 \\
\hline Female, n (\%) & $88(62)$ & $88(62)$ & $>0.99$ \\
\hline $\mathrm{BMI}, \mathrm{kg} / \mathrm{m}^{2}$, mean $\pm \mathrm{SD}$ & $28.6 \pm 6.5$ & $27.7 \pm 5.4$ & 0.37 \\
\hline Smoking status, n (\%) & & & 0.62 \\
\hline Never & $46(32)$ & $53(37)$ & \\
\hline Former & $66(46)$ & $59(41)$ & \\
\hline Current & $31(22)$ & $31(22)$ & \\
\hline \multicolumn{4}{|l|}{ RA characteristics } \\
\hline $\mathrm{RF} /$ anti-CCP positivity, $\mathrm{n}(\%)$ & $96(68)$ & $94(66)$ & 0.80 \\
\hline $\begin{array}{l}\text { Year of RA diagnosis, } \\
\text { mean } \pm \text { SD }\end{array}$ & $1993 \pm 8.6$ & $1994 \pm 8.6$ & 0.33 \\
\hline \multicolumn{4}{|l|}{ CVD risk factors, $\mathrm{n}(\%)$} \\
\hline Hypertension & $81(57)$ & $56(39)$ & 0.003 \\
\hline Diabetes mellitus & $22(15)$ & $11(8)$ & 0.04 \\
\hline Dyslipidemia & $91(64)$ & $88(62)$ & 0.71 \\
\hline Lipid-lowering drugs & $26(18)$ & $24(17)$ & 0.76 \\
\hline
\end{tabular}

CCP: cyclic citrullinated peptide antibody; CVD: cardiovascular disease; HF: heart failure; RA: rheumatoid arthritis; RF: rheumatoid factor.
Table 2. Heart failure characteristics in the HF group based on the Framingham criteria.

\begin{tabular}{lc}
\hline & $\begin{array}{c}\text { Subjects Who Fulfilled } \\
\text { the Criteria, n/N }(\%)\end{array}$ \\
\hline Major criteria & \\
Paroxysmal nocturnal dyspnea or orthopnea & $26 / 113(23)$ \\
Elevated jugular venous pressure & $78 / 119(66)$ \\
Pulmonary rales & $121 / 139(87)$ \\
Cardiomegaly on chest radiograph & $80 / 132(61)$ \\
Pulmonary edema on chest radiograph & $88 / 133(66)$ \\
Third heart sound & $15 / 121(12)$ \\
Hepatojugular reflux & $14 / 111(13)$ \\
Minor criteria & \\
Ankle edema & $109 / 136(80)$ \\
Nocturnal cough & $18 / 112(16)$ \\
Dyspnea on exertion & $122 / 139(88)$ \\
Hepatomegaly & $3 / 115(3)$ \\
Pleural effusion & $80 / 132(61)$ \\
Tachycardia (heart rate $>120$ beats/min) & $7 / 142(5)$ \\
\hline
\end{tabular}

Fulfillment of the Framingham criteria for HF required the presence of 2 major or 1 major and 2 minor criteria. HF: heart failure.

Table 3. HCQ use prior to index date/HF diagnosis in patients with RA by HF status.

\begin{tabular}{lccc}
\hline & $\begin{array}{c}\text { HF, } \\
\mathrm{n}=71\end{array}$ & $\begin{array}{c}\text { Non-HF, } \\
\mathrm{n}=69\end{array}$ & $P$ \\
\hline $\begin{array}{c}\text { Cumulative duration of } \\
\text { HCQ use, yrs, median (IQR) }\end{array}$ & $2.8(0.6-10)$ & $2.5(0.7-8.2)$ & \\
$\begin{array}{c}\text { Years from first ever HCQ start } \\
\text { to index date, median (IQR) }\end{array}$ & $9.9(5.4-16.9)$ & $8.2(3.8-15.0)$ & 0.26 \\
$\begin{array}{c}\text { HCQ cumulative dose, g, } \\
\text { median (IQR) }\end{array}$ & $371(71-1159)$ & $302(89-1043)$ & 0.98 \\
$\begin{array}{c}\text { Patients with HCQ cumulative } \\
\text { dose } \geq 300 \mathrm{~g}, \mathrm{n}(\%)\end{array}$ & $39(55)$ & $37(54)$ & 0.88 \\
\hline
\end{tabular}

HCQ: hydroxychloroquine; HF: heart failure; RA: rheumatoid arthritis.

logistic models showed that duration of HCQ use prior to index was not associated with increased risk of developing HF (OR 0.98, 95\% CI 0.91-1.05). The most recent HCQ discontinuation prior to HF diagnosis/index date was reported to be due to adverse effects in 11 (24\%) of $46 \mathrm{HF}$ patients who had any prior discontinuation of HCQ and 10 (22\%) of 45 non-HF patients who had any prior discontinuation of HCQ. Retinal toxicity prior to index date was comparable in both HF and non-HF cases and controls, being $4 \%$ and $9 \%$, respectively $(P=0.27)$.

HCQ dose and risk of HF. Table 3 shows HCQ characteristics of patients that received HCQ prior to index date. The median (IQR) cumulative dose in patients receiving HCQ prior to index date/HF diagnosis was 371 (71-1159) g and 302 (89-1043) g, respectively. Of the HF cases, $55 \%$ received a cumulative dose of $\geq 300 \mathrm{~g}$ compared to $54 \%$ in non-HF controls. The HCQ cumulative dose was not associated with HF (OR 0.96, 95\% CI 
$0.90-1.03$ per $100 \mathrm{~g}$ ). Likewise, no statistically significant association was found for patients with a cumulative dose $\geq 300 \mathrm{~g}$ compared to those with $<300 \mathrm{~g}$, although the $95 \% \mathrm{CI}$ was wide (OR 0.92, 95\% CI 0.41-2.08).

\section{DISCUSSION}

In light of the recent global interest in HCQ being considered a potential drug for treatment and/or prophylaxis of coronavirus disease 2019 (COVID-19), CV safety of HCQ has been a subject of increasing interest. ${ }^{10}$ To our knowledge, this is the first population-based study to examine the association between chronic HCQ use and HF in patients with incident RA.

Since its approval by the U.S. Food and Drug Administration in 1955, HCQ has been widely used in the treatment of RA, systemic lupus erythematosus (SLE), and other rheumatic diseases. ${ }^{4,11}$ HCQ is believed to exert an immunomodulatory effect by its ability to interfere with the maturation of autophagosomes. Additionally, it hinders the process of antigen presentation and lymphocytic activity, the same mechanism that could explain the drug's known adverse effects. ${ }^{12}$

The inflammatory burden characteristic of RA has been linked to the increased risk of $\mathrm{CV}$ disease, including $\mathrm{HF}^{3,13} \mathrm{HF}$ development in patients with RA is multifactorial, with contribution from CV risk factors and RA disease-related factors. ${ }^{3}$ The relationship between HCQ and HF has been controversial, with one argument suggesting a cardioprotective effect of $\mathrm{HCQ}$, while the counterargument supports a cardiotoxic role. In a metaanalysis conducted to associate the use of HCQ and chloroquine (CQ) in rheumatic patients (RA, SLE, lupus nephritis) with CV risk, Liu, et al reported a reduced risk of CV disease development in HCQ users. ${ }^{14}$

The proposed spectrum of cardiotoxic effects of HCQ include conduction disorders, restrictive cardiomyopathy, left ventricular hypertrophy, ventricular dysfunction, and valvular abnormalities. ${ }^{5,15}$ Although this evidence is based on case reports/series and drug surveillance reports rather than population-based longitudinal data, it is supported by 2 main rationales. First, HCQ cardiotoxicity has been characterized for its specific histological appearance on endomyocardial biopsy (EMB). ${ }^{16}$ Second, improvement after withdrawal of HCQ has been reported both clinically and histologically. ${ }^{17,18}$

The standard dose of HCQ in patients with RA ranges from 200 to $400 \mathrm{mg} /$ day, and the treatment is long term. Assessment of the risk of HF depending on cumulative dose of HCQ showed no statistically significant associations for cumulative doses assessed as a continuous variable or using a cut-off of $\geq 300 \mathrm{~g}$. However, the association for the cumulative dose $\geq 300 \mathrm{~g}$ had a wide CI (OR 0.92, 95\% CI 0.41-2.08), including both potentially meaningful benefit and harm.

The risk of retinopathy associated with HCQ has been characterized as a cumulative dose of $1000 \mathrm{~g}$ over a period of use of 5-7 years. ${ }^{19}$ While retinal toxicity associated with HCQ use was not the primary question of this study, retinal toxicity prior to index date was comparable in both HF and non-HF groups.

With many reviews analyzing the cardiotoxicity of $\mathrm{HCQ}$ and CQ in combination, this work focuses only on HCQ. ${ }^{5,15} \mathrm{HCQ}$ has been known to be less toxic in comparison to $\mathrm{CQ}$, hence is used more frequently today. ${ }^{20}$ Further, this population of only RA naturally excludes other rheumatology patients receiving HCQ, particularly patients with SLE in whom published reports on HF have been more predominant. In a recent pharmacovigilance analysis, Goldman, $e t$ a $l^{10}$ showed association of HCQ/ CQ use with HF (reporting OR 2.2). This risk was largely driven by persons with SLE and Sjögren syndrome compared with all other users, including those with RA.

There are several limitations to this study. First, as with any retrospective study, only information available from medical records was used to ascertain exposures and outcomes. However, availability of complete medical records from all healthcare providers in the area and standardized case ascertainment likely minimized this bias. Second, no patient follow-up after HCQ discontinuation was performed nor was EMB available in our medical records. Third, the population size, although the largest to date, still limited the study. Fourth, the prevalence of HTN and DM was higher in patients with $\mathrm{HF}$ as compared to those without HF. While both HTN and DM can affect the risk of $\mathrm{HF}$, this imbalance in baseline characteristics between patients with and without $\mathrm{HF}$ is unlikely to have major implications on the main results of the study, as no statistically significant association between HCQ use and risk of HF was detected. Finally, the HF diagnosis was based on retrospective chart review, and information on ejection fraction, presence of arrhythmia, biological markers of HF, and type of HF was not available. However, similar to our previous studies, all patients with HF fulfilled the Framingham criteria, which have been shown to have high sensitivity (92\%) for HF diagnosis. ${ }^{21}$

In conclusion, this is the first population-based study to quantify the association between HCQ use and HF in patients with RA, to our knowledge. We found no increased risk of HF in this RA population receiving HCQ. Larger prospective studies are needed to define the safety of higher cumulative doses of HCQ with regard to HF development, as well as to identify the incidence of HCQ-related cardiotoxicity in patients with RA, in order to define the subgroups of high-risk patients and the need for $\mathrm{CV}$ screening.

\section{REFERENCES}

1. Smolen JS, Aletaha D, McInnes IB. Rheumatoid arthritis. Lancet 2016;388:2023-38.

2. Aviña-Zubieta JA, Choi HK, Sadatsafavi M, Etminan M, Esdaile JM, Lacaille D. Risk of cardiovascular mortality in patients with rheumatoid arthritis: a meta-analysis of observational studies. Arthritis Care Res 2008;59:1690-7.

3. Nicola PJ, Maradit-Kremers H, Roger VL, Jacobsen SJ, Crowson $\mathrm{CS}$, Ballman KV, et al. The risk of congestive heart failure in rheumatoid arthritis: a population-based study over 46 years. Arthritis Rheum 2005;52:412-20.

4. Schrezenmeier E, Dörner T. Mechanisms of action of hydroxychloroquine and chloroquine: implications for rheumatology. Nat Rev Rheumatol 2020;16:155-66.

5. Tönnesmann E, Kandolf R, Lewalter T. Chloroquine cardiomyopathy - a review of the literature. Immunopharmacol Immunotoxicol 2013;35:434-42.

6. Kremers HM, Myasoedova E, Crowson CS, Savova G, Gabriel SE, 
Matteson EL. The Rochester Epidemiology Project: exploiting the capabilities for population-based research in rheumatic diseases. Rheumatology 2011;50:6-15.

7. Arnett FC, Edworthy SM, Bloch DA, McShane DJ, Fries JF, Cooper NS, et al. The American Rheumatism Association 1987 revised criteria for the classification of rheumatoid arthritis. Arthritis Rheum 1988;31:315-24.

8. Ho KKL, Pinsky JL, Kannel WB, Levy D. The epidemiology of heart failure: the Framingham Study. J Am Coll Cardiol 1993;4 Suppl A:6A-13A.

9. Myasoedova E, Crowson CS, Matteson EL, Davis JM, Therneau TM, Gabriel SE. Decreased cardiovascular mortality in patients with incident rheumatoid arthritis (RA) in recent years: dawn of a new era in cardiovascular disease in RA? J Rheumatol 2017;44:732-9.

10. Goldman A, Bomze D, Dankner R, Hod H, Meirson T, Boursi B, et al. Cardiovascular adverse events associated with hydroxychloroquine and chloroquine: a comprehensive pharmacovigilance analysis of pre-COVID-19 reports. Br J Clin Pharmacol 2021;87:1432-42.

11. Singh JA, Furst DE, Bharat A, Curtis JR, Kavanaugh AF, Kremer JM, et al. 2012 Update of the 2008 American College of Rheumatology (ACR) Recommendations for the use of disease-modifying anti-rheumatic drugs and biologics in the treatment of rheumatoid arthritis (RA). Arthritis Care Res 2012;64:625-39.

12. Frustaci A, Morgante E, Antuzzi D, Russo MA, Chimenti C. Inhibition of cardiomyocyte lysosomal activity in hydroxychloroquine cardiomyopathy. Int J Cardiol 2012;157:117-9.

13. Crowson CS, Nicola PJ, Kremers HM, O'Fallon WM, Therneau TM, Jacobsen SJ, et al. How much of the increased incidence of heart failure in rheumatoid arthritis is attributable to traditional cardiovascular risk factors and ischemic heart disease? Arthritis Rheum 2005;52:3039-44.

14. Liu D, Li X, Zhang Y, Kwong JS-W, Li L, Zhang Y et al. Chloroquine and hydroxychloroquine are associated with reduced cardiovascular risk: a systematic review and meta-analysis. Drug Des Devel Ther 2018;12:1685-95.

15. Chatre C, Roubille F, Vernhet H, Jorgensen C, Pers Y-M. Cardiac complications attributed to chloroquine and hydroxychloroquine: a systematic review of the literature. Drug Saf 2018;41:919-31.

16. Ratliff NB, Estes ML, Myles JL, Shirey EK, McMahon JT. Diagnosis of chloroquine cardiomyopathy by endomyocardial biopsy. N Engl J Med 1987;316:191-3.

17. Cotroneo J, Sleik KM, Rene Rodriguez E, Klein AL. Hydroxychloroquine-induced restrictive cardiomyopathy. Eur J Echocardiogr 2007;8:247-51.

18. Joyce E, Fabre A, Mahon N. Hydroxychloroquine cardiotoxicity presenting as a rapidly evolving biventricular cardiomyopathy: key diagnostic features and literature review. Eur Heart J Acute Cardiovasc Care 2013;2:77-83.

19. Wolfe F, Marmor MF. Rates and predictors of hydroxychloroquine retinal toxicity in patients with rheumatoid arthritis and systemic lupus erythematosus. Arthritis Care Res 2010;62:775-84.

20. Aviña-Zubieta J, Galindo-Rodriguez G, Newman S, Suarez-Almazor $\mathrm{M}$, Russell A. Long term effectiveness of antimalarial drugs in rheumatic diseases. Ann Rheum Dis 1998;57:582-7.

21. Maestre A, Gil V, Gallego J, Aznar J, Mora A, Martín-Hidalgo A. Diagnostic accuracy of clinical criteria for identifying systolic and diastolic heart failure: cross-sectional study. J Eval Clin Pract 2009;15:55-61. 